\title{
Immobilized Particles in Gel Matrix-Type Porous Media. Nonhomogeneous Cell Distribution
}

\author{
Manuel Mota,* J osé A. Teixeira, and Alexander Yelshin
}

Centro de Eng. Biológica - IBQF, University of Minho, Campus de Gualtar, 4710-057 Braga, Portugal

\begin{abstract}
The conventional random pore model assumes a homogeneous cell distribution in the gel matrix used to immobilize cells. However, the validity of this model is restricted to values of the exponent $\alpha$, between 1.8 and 2.25, of a model power function relating the diffusivity coefficient in the matrix with the overall cell volume fraction in the system. Based on the analysis of published data for diffusion in gels with immobilized cells and on the homogeneous approach for the random pore model developed in a previous work, a new, nonhomogeneous approach is proposed for $\alpha$ values outside the range 1.8-2.25. To explain these data, two main types of nonhomogeneous cell distribution were considered: (1) nonhomogeneous cell distribution in the gel for $\alpha>$ 2.25 (type 1) and (2) nonhomogeneity related with anisotropy of cell space orientation when $\alpha<1.8$ (type 2). In the case of nonhomogeneity of type 1 , the cell volume fraction in the layers occupied by cells must be considered in place of the concept previously used for homogeneous distribution, viz., the average cell volume fraction. This model underlines that accumulation of cells in a thin layer close to the surface improves their nutrient intake. For nonhomogeneity of type 2, the tortuosity of such a system is smaller than should be expected if spherical cells were considered, thereby changing the effective diffusion. The model proposed in this work proved to fit into several real cases reported in the literature.
\end{abstract}

\section{Introduction}

There has been an increasing interest on the use of immobilized cells and enzymes in environmental, pharmaceutical, food, and biotechnologies.

To increase the overall productivity of immobilized cell systems, a complete characterization of the phenomena associated with the immobilization process is required. Mass transfer limitations have been reported as a main drawback of these systems, causing a reduction in the overall reaction rate as a consequence of the reduced rate of transport of nutrients inside the immobilization matrix.

In a previous work (1), diffusion in pure gels and gels with immobilized cells was analyzed and a model describing diffusion in a gel with immobilized cells was developed and validated. The dependence of the diffusivity coefficient $\eta=\mathrm{D}_{\mathrm{e}} / \mathrm{D}_{0}$ on cell volume fraction $\phi_{\mathrm{c}}$ for a gel with immobilized cells was described as:

$$
\eta=\eta_{\mathrm{g}} \eta_{\mathrm{c}}=\frac{\epsilon_{\mathrm{g}}}{\mathrm{T}_{\mathrm{g}}} \cdot \frac{\epsilon_{\mathrm{c}}}{\mathrm{T}_{\mathrm{c}}\left(\epsilon_{\mathrm{c}}\right) \mathrm{T}_{\mathrm{g}}\left(\epsilon_{\mathrm{c}}\right)}
$$

where

$$
\eta_{\mathrm{c}}=\frac{\mathrm{D}_{\mathrm{e}}}{\mathrm{D}_{\mathrm{g}}} \text {, or } \mathrm{D}_{\mathrm{e}}=\eta_{\mathrm{c}} \mathrm{D}_{\mathrm{g}} \text { and } \frac{\mathrm{D}_{\mathrm{e}}}{\mathrm{D}_{0}}=\eta_{\mathrm{c}}\left(\mathrm{D}_{\mathrm{g}} / \mathrm{D}_{0}\right)=\eta_{\mathrm{c}} \eta_{\mathrm{g}}
$$

$D_{e}$ is the effective diffusion coefficient in gel with immobilized cells; $D_{g}$ is the diffusion coefficient in pure gel;

* To whom correspondence should be addressed. Ph: +351253604400. E-mail: mmota@deb.uminho.pt.
$D_{0}$ is the diffusion coefficient in bulk solution; $\eta_{\mathrm{g}}=\mathrm{D}_{\mathrm{g}} /$ $\mathrm{D}_{0}=\epsilon_{\mathrm{g}} / T_{\mathrm{g}}$ is the partial diffusi vity coefficient in gel; $\eta_{\mathrm{c}}$ is the partial diffusivity coefficient in immobilized cells structure; $\epsilon_{\mathrm{g}}=1-\phi_{\mathrm{p}}$ is the gel porosity; $\phi_{\mathrm{p}}$ is the polymer volume fraction in gel; $T_{g}$ is the pure gel tortuosity (average ratio of molecule path to porous media thickness); $T_{\mathrm{g}}\left(\epsilon_{\mathrm{c}}\right)$ is the tortuosity of gel matrix filled by cells (small scal e tortuosity), and $T_{c}\left(\epsilon_{c}\right)$ is the tortuosity created in the matrix by cells presence (large scale tortuosity).

By introducing a complex tortuosity value, $\mathrm{T}_{\mathrm{c}}\left(\epsilon_{\mathrm{c}}\right)=1 / \epsilon^{\beta}$ and $\mathrm{T}_{\mathrm{g}}\left(\epsilon_{\mathrm{c}}\right)=1 / \epsilon^{\gamma}$, where $\beta$ and $\gamma$ are order values $(\beta<1$, $\gamma<1$ ), eq 1 can be written as

$$
\begin{gathered}
\eta=\mathrm{D}_{\mathrm{e}} / \mathrm{D}_{0}=\eta_{\mathrm{g}}\left(1-\phi_{\mathrm{c}}\right)\left(1-\phi_{\mathrm{c}}\right)^{\beta}\left(1-\phi_{\mathrm{c}}\right)^{\gamma}= \\
\eta_{\mathrm{g}}\left(1-\phi_{\mathrm{c}}\right)^{\alpha} \\
\alpha=1+\beta+\gamma
\end{gathered}
$$

This model, developed assuming a homogeneous cell distribution in the gel, was shown to fit with high accuracy to experimental data.

Nevertheless, not all experimental data could be explained on the basis of this model, particularly when anomalously small or large values of the diffusion coefficient $D_{d} / D_{0}$ vs $\phi_{c}$ were measured. These cases must be considered assuming a nonhomogeneous cell distribution inside the gel matrix and will be the subject of the following analysis.

Two main types of nonhomogeneous cell distribution based on the random pore model will be considered below: (1) nonhomogeneous cells or cells cluster distribution in a gel (type 1 nonhomogeneity) and (2) nonhomogeneity related with anisotropy of cells space orientation (type 2 nonhomogeneity). 
Nonhomogeneous cell distributions have been described in different immobilized cells applications. For penicillin production, Behie and Gaucher (2) showed that cell growth occurs mainly near the surface of the carrageenan beads (type 1 nonhomogeneity); the microphotograph presented by the authors also demonstrates that the cell's main axis is preferentially oriented along the bead's radial direction (type 2 nonhomogeneity). Arnaud and Lacroix (3) showed the existence of a high cell density layer near the bead surface, concluding that the depth of the cell layer depends on the fermentation conditions and on physical parameters such as the bead diameter, the kind of substrate, and the product diffusion coefficients. De Backer et al. (4) noted the same effect, and Hannoun and Stephanopoulos (5) found that, instead of being well distributed throughout the gel, immobilized growing cells are found in clumps forming dense layers near the gel surface. They al so showed that the presence of $20 \%$ of dead yeast cells had no effect on solute diffusivities.

Westrin and Axelsson (6) compared some published experimental data that quantitatively express the effective diffusion coefficient as a function of $\phi_{c}$, the cell volume fraction, using well-known equations developed for mass transfer in heterogeneous media. Some of the data presented anomalous high or small effective diffusion coefficients, exceeding the bounds of the applied models. In the first part of their work, the authors have shown that the homogeneous model (eq 2) could describe the data by admitting values for $\alpha$ considerably larger or smaller than 2.0, which in turn reduces the physical meaning of the $\alpha$ parameter.

Korgel et al. (7) mentioned that the random pore model predicts with high accuracy, in most of the situations, sugar effective diffusivity in gels with immobilized cells. Neverthel ess, several data from the literature do not fit into the applied models, and the reported experimental values are clearly outside the $95 \%$ confidence limits for $\alpha$.

De Backer et al. (4) used for the interpretation of experimental data on glucose diffusion in immobilized yeasts a value of 1.0 for $\alpha$, meaning that tortuosity equals 1.0 if the relationship $D_{e} / D_{0}=\epsilon / T$ is used ( $T$ stands for the total heterogeneous system tortuosity).

In some cases (Riley et al. $(8,9)$, Westrin and Axelsson (6), Libicki et al. (10), Chresand et al. (11), Ho and J u (12)), the Maxwell's model, as described in eq 4 for the diffusion in gels with immobilized cells, is applied for the description and the explanation of high effective diffusion coefficients $(\alpha<2)$ :

$$
\frac{D_{e}}{D_{0}}=\frac{2 D_{0}+D_{c}-2 \phi_{c}\left(D_{0}-D_{c}\right)}{2 D_{0}+D_{c}+\phi_{c}\left(D_{0}-D_{c}\right)}
$$

where $D_{0}$ is the diffusion coefficient of a given species through the continuous phase, and $D_{c}$ is the diffusion coefficient through the dispersed phase.

According to this model, the cells are considered as a permeable phase and ratio $D_{d} D_{0}$ is assumed to be in the range $0 \leq D_{d} / D_{0}<1$. N onpermeable cells correspond to $\mathrm{D}_{d} \mathrm{D}_{0}=0$. Living cells have specific permeability functions for substrate and cannot be considered as a permeable unit similar to a membrane as in membrane separation processes. This means that other reasons must be found elsewhere for explaining observed high effective diffusions in some immobilized cell systems.

By assuming a nonhomogeneous model in the present work, we shall try to explain the experimental diffusion discrepancies observed in several cases reported in the literature.
1. Nonhomogeneous Cells or Cells Cluster Distribution in Gel (Type 1). An explanation to the extreme values of $\alpha>2.25$ that were determined in the experiments discussed above will be proposed in the following pages.

When cells are concentrated near the bead (membrane) surface it means that a gradient of volume fraction of cells inside the gel matrix occurs. To study this problem, a set of conditions will be postulated.

In the first place, a gel membrane with rod-shaped immobilized cells and with an average volume fraction of cells $\phi_{c}$ will be assumed. As it has been shown that in these systems cells tend to concentrate near the membrane surface, two gel layers will be considered. The first layer will contain most of the cells, whereas the second layer will exhibit a low or very low cell concentration, assumed to be zero for model development. Behie and Gaucher (2) showed the presence of this type of cell distribution in gel beads used in a three-phase fluidized bed bioreactor.

Using mass transfer coefficients, the ratio $D_{e} / D_{g}$ for the two layers system can be calculated. The total mass transfer coefficient, $k$, is determined by the equation

$$
\frac{1}{\mathrm{k}}=\frac{1}{\mathrm{k}_{1}}+\frac{1}{\mathrm{k}_{2}}
$$

where $k_{1}$ is the mass transfer coefficient for layer with immobilized cells, and $\mathrm{k}_{2}$ is the mass transfer coefficient for the second layer with low cell concentration.

The mass transfer coefficient in an elementary layer i can be written as

$$
\mathrm{k}_{\mathrm{i}}=\frac{\mathrm{D}_{\mathrm{ei}}}{\mathrm{L}_{\mathrm{i}}}=\frac{\mathrm{D}_{\mathrm{g}} \eta_{\mathrm{i}}}{\mathrm{L}_{\mathrm{i}}}
$$

where $D_{e i}$ is the effective diffusion coefficient for the $i$ layer, $L_{i}$ is the thickness of the $i$ layer. $\eta_{i}$ is the ratio $\epsilon_{\mathrm{i}} / T_{\mathrm{i}}=\left(1-\phi_{\mathrm{c}}\right) / T_{\mathrm{i}}=\mathrm{D}_{\mathrm{e} i} / \mathrm{D}_{\mathrm{g}}$, where $\epsilon_{\mathrm{i}}=\left(1-\phi_{\mathrm{c}}\right)$ is the porosity of the i layer; $\phi_{\mathrm{c}}$ is the volume fraction of cells in the $i$ layer; and $T_{i}$ is the tortuosity of the $i$ layer.

Hence, when we consider the occurrence of two layers, the mass transfer coefficient is

$$
\frac{1}{\mathrm{k}}=\frac{\mathrm{L}}{\mathrm{D}_{\mathrm{g}} \eta}=\frac{\mathrm{L}_{1}}{\mathrm{D}_{\mathrm{g}} \eta_{\mathrm{c} 1}}+\frac{\mathrm{L}_{2}}{\mathrm{D}_{\mathrm{g}} \eta_{\mathrm{c} 2}}=\frac{1}{\mathrm{D}_{\mathrm{g}}}\left(\frac{\delta \cdot \mathrm{L}}{\eta_{\mathrm{c} 1}}+\frac{(1-\delta) \mathrm{L}}{\eta_{\mathrm{c} 2}}\right)
$$

where $L=L_{1}+L_{2}$ is the membrane thickness, and $\delta=$ $\mathrm{L}_{1} / \mathrm{L}$.

From eq 7 we have

$$
\frac{1}{\eta}=\frac{\delta}{\eta_{\mathrm{c} 1}}+\frac{1-\delta}{\eta_{\mathrm{c} 2}}
$$

where $\eta_{\mathrm{c}}=\eta_{\mathrm{g}}\left(1-\phi_{\mathrm{c}}\right)^{\alpha}$ is the diffusivity determined by eq 2.

After rearrangement of eq 8 , we have

$$
\eta=\left(1-\phi_{\mathrm{c}}\right)^{\alpha}=\frac{\eta_{\mathrm{c} 1} \eta_{\mathrm{c} 2}}{\eta_{\mathrm{c} 2} \delta+\eta_{\mathrm{c} 1}(1-\delta)}
$$

where $\alpha$ is the parameter associated with the homogeneous model.

Assuming a homogeneous cell distribution for each layer and $\alpha=2$, as for the random pore model, eq 9 may 
be written as

$$
\eta=\left(1-\phi_{\mathrm{c}}\right)^{\alpha}=\frac{\left[\left(1-\phi_{\mathrm{c} 1}\right)\left(1-\phi_{\mathrm{c} 2}\right)\right]^{2}}{\delta\left(1-\phi_{\mathrm{c} 2}\right)^{2}+(1-\delta)\left(1-\phi_{\mathrm{c} 1}\right)^{2}}
$$

and a more compact equation, in terms of porosity, is obtained:

$$
\eta=\epsilon^{\alpha}=\frac{\left(\epsilon_{\mathrm{c} 1} \epsilon_{\mathrm{c} 2}\right)^{2}}{\delta \epsilon_{\mathrm{c} 2}^{2}+(1-\delta) \epsilon_{\mathrm{c} 1}^{2}}
$$

The average porosity of the bilayer system is cal culated as

$$
\epsilon=\left(1-\phi_{\mathrm{c}}\right)=\delta \epsilon_{\mathrm{c} 1}+(1-\delta) \epsilon_{\mathrm{c} 2}
$$

Using eqs 10 and 11, we can estimate the value of $\alpha$ as (9)

$$
\begin{gathered}
\alpha=\frac{\ln \left\{\frac{\left(\epsilon_{\mathrm{c} 1} \epsilon_{\mathrm{c} 2}\right)^{2}}{\delta \epsilon_{\mathrm{c} 2}^{2}+(1-\delta) \epsilon_{\mathrm{c} 1}^{2}}\right\}}{\ln \left[\delta \epsilon_{\mathrm{c} 1}+(1-\delta) \epsilon_{\mathrm{c} 2}\right]} \\
\delta \leq \frac{\epsilon_{\mathrm{c} 2}}{\epsilon_{\mathrm{c} 2}-\epsilon_{\mathrm{c} 1}}
\end{gathered}
$$

for $\delta=1$ and $\delta=0, \alpha=2$.

Simplified examples of the model are shown in Figures 1-3. Assuming that the first layer contains all of the immobilized cells and that the second layer is free of cells, then $\eta_{\mathrm{c} 2}=1$ for a pure gel and eq 9 can be simplified to

$$
\mathrm{D}_{\mathrm{e}} / \mathrm{D}_{\mathrm{g}}=\frac{\left(1-\phi_{\mathrm{cl}}\right)^{\alpha}}{\delta+\left(1-\phi_{\mathrm{c} 1}\right)^{\alpha}(1-\delta)}
$$

In Figure 3 we represent the situation corresponding to a large value of $\alpha$ when the average porosity (or volume fraction) of immobilized cells is used. The real dependence of $D_{e} / D_{g}$ on $\phi_{c 1}$ (eq 13) is also shown.

Whenever we have a nonhomogeneous cell distribution in a gel and we try to fit the homogeneous model, the value of $\alpha$ must be much larger than 2, which has no physical meaning for this type of porous media.

In turn, if we assume the existence of two gel layers, then the values for $\alpha$ calculated from eq 13 become smaller and more reasonable. In other words, this is the main reason for the large values of $\alpha$ when the homoge neous model of cells distribution in gel is applied to a nonhomogeneous distribution.

Validation of this model requires an in depth knowledge of biomass distribution in immobilization matrixes. Although some authors, as previously mentioned, have described the occurrence of higher biomass concentrations near the gel surface, no accurate data were available describing biomass distribution throughout the immobilization matrix. This aspect emphasizes the urgent need of experimental data on the characterization of cell distribution in immobilized cell systems.

2. Nonhomogeneity Related with Anisotropy of Cell Space Orientation (Type 2). To explain the occurrence of values of $\alpha<1.8$ in the case of the homogeneous model, a nonhomogeneous cell distribution in the gel may be assumed. This effect can be related with a possible nonspherical shape of cells; they may be ellipsoidal, rodlike, etc. Nonspherical overlapped cell

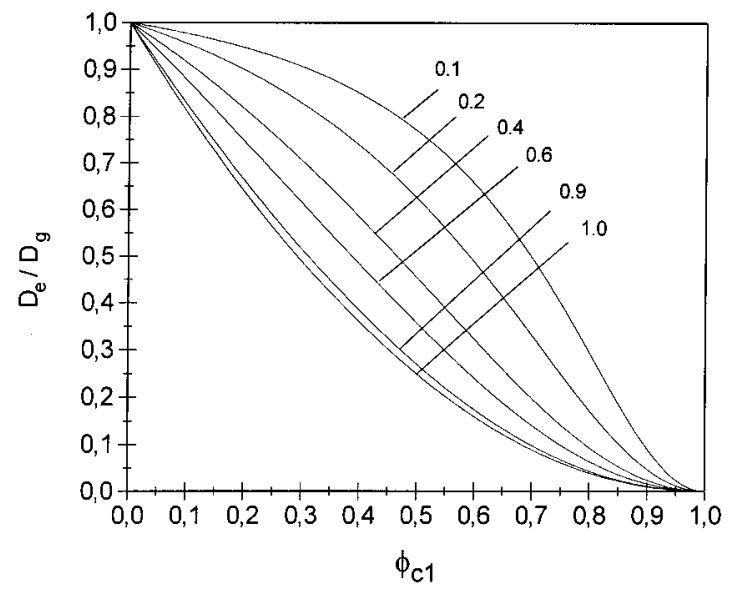

Figure 1. Variation of $D_{e} / D_{g}$ with $\phi_{c 1}$ (see eq 13) for different thickness values $\delta$ of the layer with immobilized cells, $\alpha=2.0$.

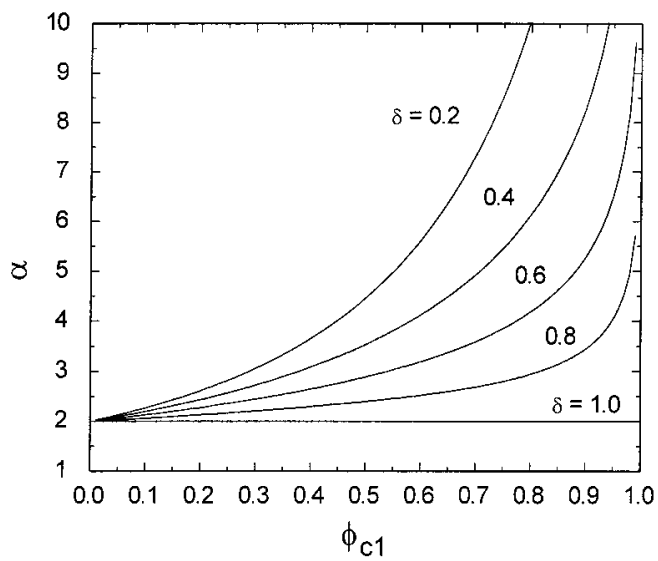

Figure 2. Variation of $\alpha$ with $\phi_{\mathrm{c} 1}$ for different values of $\delta$ of the immobilized cell layer.

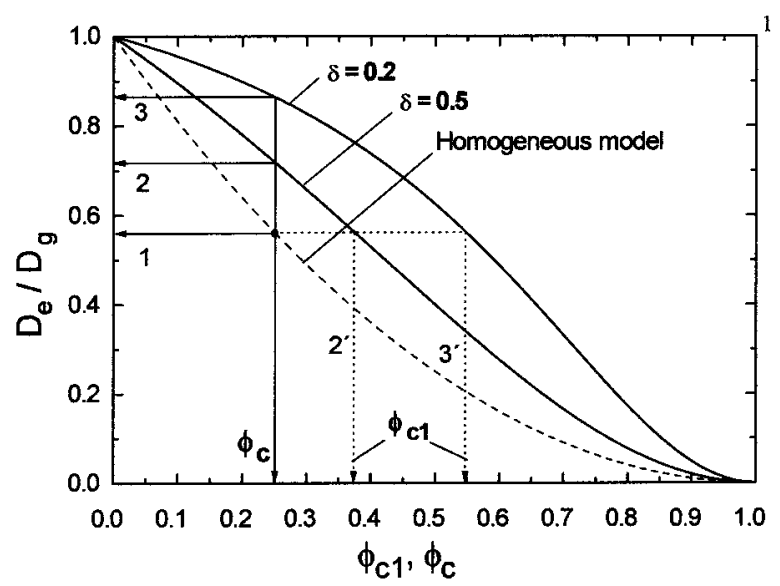

Figure 3. Dependence of $D_{e} / D_{g}$ on $\phi_{c 1}$ (eq 13, for $\delta=0.2, \alpha=$ 2.0 and for $\delta=0.5, \alpha=2.0$ ). The values of $\delta$ (cell layer thickness) are marked on the graph. For any value of the overall cell fraction $\phi_{c}$ accumulation of cells in a thin outer layer is a biological advantage as compared to an even cell distribution (effective diffusivity is higher).

clusters can al so be considered as asymmetric bodies. F or example, Riley et al. $(8,9)$, in a computer simulation of cells distribution with arbitrarily overlapping positions, obtained asymmetric clusters, which have a much greater length in one direction than along another direction. The phenomenon takes place when, instead of a random distribution of cells or cell clusters, we have some kind of preference for a defined cell orientation. A schematic 


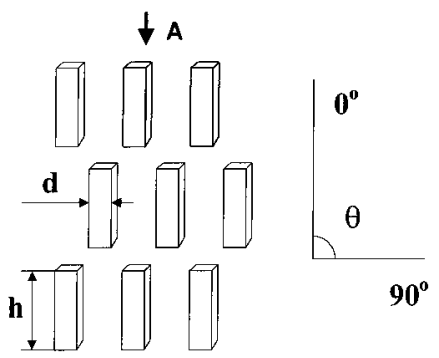

Figure 4. Graphs of cells or cell clusters oriented in one direction represented in a prismatic simplified form, where $d$ and $\mathrm{h}$ are the width and length of the prism, respectively. $\mathrm{A}=$ flow direction.

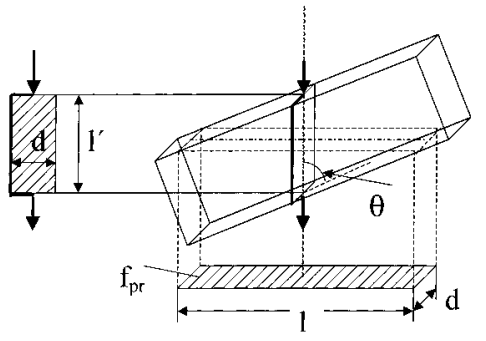

(a)

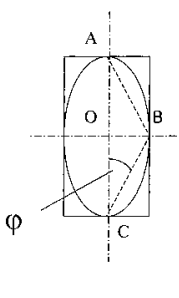

(b)
Figure 5. (a) Schema to determine the horizontal projection area $f_{\mathrm{pr}}$ and a pathway (arrows) tortuosity around a prism inclined under angle $\theta$ to the vertical direction. Hatched rectangles in the scheme are the cross-section areas, where I and I' are the length of each cross-section. (b) Schema of simplified calculation of the tortuosity around cylinder inscribed to the prism: $T=1 / \cos (\varphi)$, where $\varphi=f(\theta)$. Calculation of tortuosity was done according to the classical definition of Bear (13).

representation of fully oriented cells may be seen in Figure 4.

In the type 2 nonhomogeneity, two effects must be taken into consideration. On one hand, we may obtain different free cross-section areas for different flow directions. On the other hand, the tortuosity can be smaller than should be expected for spherical cells.

Cross-Section Free Area. In the case of a regular nonspherical cell arrangement, the average porosity and, hence, the average cross-section free area available for diffusion may differ in different directions.

Let us consider a hypothetical case when the prism (Figure 4) represents the approximation to an ellipsoidal cell of diameter $\mathrm{d}$ (prism width) and a length $\mathrm{h}=\mathrm{nd}$, where $\mathrm{n}$ is the ratio of prism length $\mathrm{h}$ to its width $\mathrm{d}$.

First, it will be interesting to analyze the variation of the prism average cross-section area on a normal surface to the flow direction (A) for a different angle of orientation $\theta$ (Figure $5 \mathrm{a}$ ). The prism cross-section area during rotation from the angle $\theta=0$ to $\theta=\pi / 2$ changes from a square to a rectangle. The projection area with length I and width $\mathrm{d}$ is $\mathrm{f}_{\mathrm{pr}}=\mathrm{dl}$.

For the prism, oriented under an angle $\theta$ to the vertical, the cross-section projection area $\mathrm{f}_{\mathrm{pr}}$ can be defined as (this relation may be also used as the first approach for a cylinder inscribed in the prism)

$$
f_{p r}=\left\{\begin{array}{l}
d^{2} / \cos (\theta), \text { for } \theta \in\left[0, \theta_{1}\right] \\
d h / \sin (\theta)=n d^{2} / \sin (\theta), \text { for } \theta \in\left[\theta_{1}, \pi / 2\right]
\end{array}\right.
$$

where angle $\theta_{1}$ corresponds to the largest cross-section area and is defined as $\theta_{1}=\arccos \left(1 / \sqrt{1+n^{2}}\right)$. With the prism in vertical position, $\theta=0, f_{p r}=d^{2}$ and, for horizontal position, $\theta=\pi / 2, \mathrm{f}_{\mathrm{pr}}=\mathrm{nd}^{2}$. The dependence



Figure 6. Dependence on $\theta$ (deg) for different ratios $n=h / d$ of the dimensionless cross-section area $I / d=f_{p r} / d^{2}$ of the prism horizontal surface at the point of symmetry.

of the dimensionless cross-section area $\mathrm{f}_{\mathrm{pr}} / \mathrm{d}^{2}=\mathrm{I} / \mathrm{d}$ on $\theta$ is shown in Figure 6.

F or further analysis, let us consider an elementary unit of the porous media in the form of a cube of size $h$ with a prism of the same length inside. This approach allows us to obtain simple relationships.

Average Void Fraction of the Unit and Free CrossSection Area. The average void fraction $\epsilon_{\mathrm{av}}$ is defined by the relation $\epsilon_{\mathrm{av}}=1-\mathrm{hd}^{2} / \mathrm{h}^{3}=1-1 / \mathrm{h}^{2}$, which coincides with the free cross-section area fraction $\epsilon_{\mathrm{ver}}^{\mathrm{S}}=$ $1-1 / \mathrm{n}^{2}$ for vertical flow. However, for cell units in the horizontal position, we have $\epsilon_{\text {hor }}^{\mathrm{S}}=1-1 / \mathrm{n}$. For $\mathrm{n}=2$, $\epsilon_{\text {ver }}^{\mathrm{S}}=0.75$ and $\epsilon_{\text {hor }}^{\mathrm{S}}=0.5$, respectively.

Free Cross-Section Area Fraction vs $\theta$. The free cross-section area fraction $\epsilon_{\alpha}^{S}$ in the point of symmetry for different orientations of the prism inside the elementary unit is

$$
\epsilon_{\alpha}^{\mathrm{S}}=\left\{\begin{array}{l}
1-1 /\left[\mathrm{n}^{2} \cos (\theta)\right], \text { for } \theta \in\left[0, \theta_{1}\right] \\
1-1 /[\mathrm{n} \sin (\theta)], \text { for } \theta \in\left[\theta_{1}, \pi / 2\right]
\end{array}\right.
$$

For instance, for $\mathrm{n}=2$ and $\theta=45^{\circ}, \epsilon_{\alpha}^{\mathrm{S}}=0.646$; for $\theta$ $=60^{\circ}, \epsilon_{\alpha}^{\mathrm{S}}=0.5$ against $\epsilon_{\mathrm{ver}}^{\mathrm{S}}=0.75$. The comparison of the free cross-area fraction $\epsilon_{\alpha}^{S}$ of prism-like particle with a sphere of the same volume $\epsilon_{\mathrm{sp}}^{\mathrm{S}}=\epsilon_{\mathrm{av}(\mathrm{sp})}$ for different volume fractions of solid $\phi_{c}$ in the elementary unit is shown in Figure 7a. In Figure $7 \mathrm{~b}$ is represented the dependence of ratio $\epsilon_{\mathrm{av}} / \epsilon_{0}^{\mathrm{S}}$ for a cylindrical approach (cylinder inscribed to the prism) on volume fraction $\phi_{c}$ for different $n=h / d$. In these figures, $\epsilon_{\mathrm{av}}$ corresponds to the average free cross-section area calculated for cylinders with an angular orientation in the range of $\theta=0-$ $\pi / 2$, and $\epsilon_{0}^{\mathrm{S}}$ corresponds to the free cross-section area for cylinders oriented with $\theta=0^{\circ}$.

A dramatic change of $\epsilon_{\alpha}^{\mathrm{S}}\left(\theta=45^{\circ}\right)$ vs average void fraction $\epsilon_{\mathrm{av}}=\epsilon_{0}^{\mathrm{S}}$ when $\theta=0^{\circ}$ for the cell volume fraction $\phi_{\mathrm{c}}>0.3$ is observed. Values of $\epsilon_{\mathrm{av}} / \epsilon_{0}^{\mathrm{S}}$ close to zero for the cylinder approach are related with the fact that the crosssection area overlapping effect for different layers was not considered. Hence, a ratio $D_{e} / D_{g}$, determined in experiment in the gel with immobilized nonspherical shape cells and plotted vs average cell volume fraction $\phi_{c}$ moves to the left side of a graph, and the curve $D_{e} / D_{g}$ vs $\phi_{\mathrm{c}}$ looks more stretched (F igure 7 , dashed lines). This means that, in this case, we must use a correction function $\mathrm{a}\left(\phi_{\mathrm{c}}\right)$ similar to ratio $\mathrm{a}\left(\phi_{\mathrm{c}}\right) \propto\left(\epsilon_{0}^{\mathrm{S}} / \epsilon_{\mathrm{av}}\right)$ in eq 1 or, 


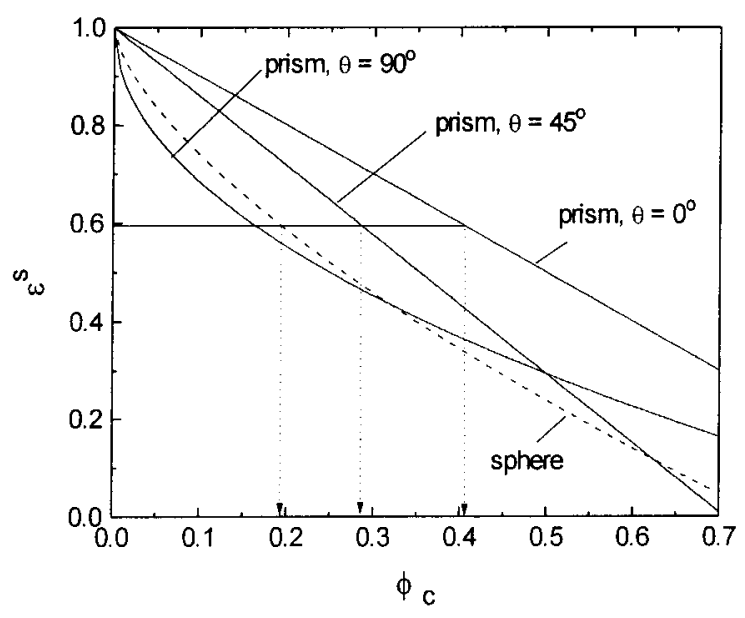

(a)

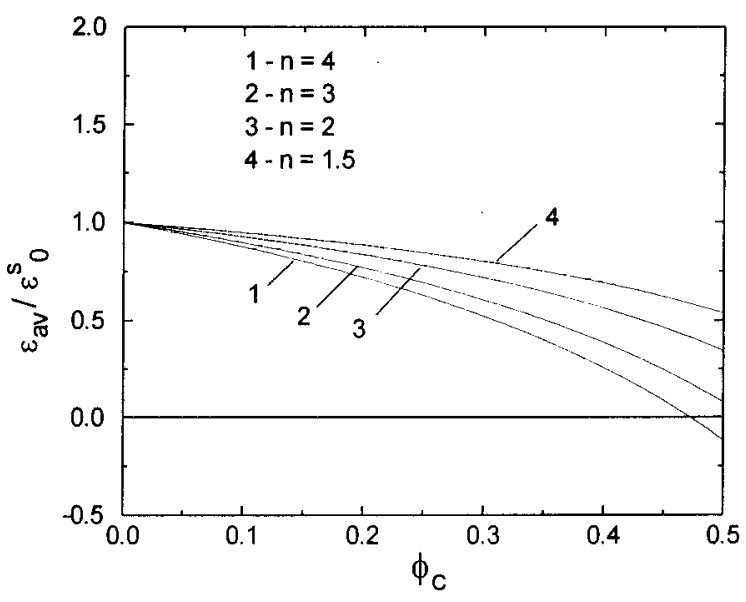

(b)

Figure 7. (a) Dependence of $\epsilon^{\mathrm{S}}$ for prism-like partide and sphere of the same volume on volume fraction of solid $\phi_{\mathrm{c}}$ in elementary unit. (b) Dependence of $\epsilon_{\mathrm{av}} / \epsilon_{0}^{\mathrm{S}}$ for cylindrical approach (cylinder inscribed to the prism) on volume fraction $\phi_{\mathrm{c}}$ for different $\mathrm{n}=\mathrm{h} / \mathrm{d}$ and uniform angular orientation in the range of $\theta=0$ to $\pi / 2$.

alternatively, use the real porosity (cross-section free area fraction) in the flow direction:

$$
\eta=\eta_{\mathrm{g}} \eta_{\mathrm{c}}=\frac{\epsilon_{\mathrm{g}}}{\mathrm{T}_{\mathrm{g}}} \cdot \frac{\mathrm{a}\left(\phi_{\mathrm{c}}\right) \cdot \epsilon_{\mathrm{c}}}{\mathrm{T}_{\mathrm{c}}\left(\epsilon_{\mathrm{c}}\right) \cdot \mathrm{T}_{\mathrm{g}}\left(\epsilon_{\mathrm{c}}\right)}
$$

Tortuosity. The above-mentioned second effect in nonhomogeneous gels is related with tortuosity $T_{c}\left(\epsilon_{c}\right)$, which has a dependence on cell volume fraction or porosity $\epsilon_{\mathrm{c}}$ other than the one for spherical cells. Tortuosity of partially oriented nonspherical cells is a complex equation and will not be considered in this article. Nevertheless, an overall estimation based on simplified assumptions may be useful.

If we assume that tortuosity of nonspherical partial oriented cells in the mass transfer direction is described by the function $T_{c}\left(\epsilon_{c}\right)=1 / \epsilon^{\beta}$, then tortuosity must stay between the following bounds: for all cells oriented vertically $\mathrm{T}_{\mathrm{c}}\left(\epsilon_{\mathrm{c}}\right) \rightarrow 1(\beta \rightarrow 0)$ with cell concentration decreasing; for all cells oriented horizontally, the tortuosity function is close to the tortuosity function for spherical cells. Hence, for the type 2 nonhomogeneity, values of $\beta$ are smaller than 0.5 , which are the corresponding values for spherical cells, and values of $\alpha$ in eq 2 must be smaller than 1.8.

An excellent example of this phenomenon can be observed in the work by Libicki et al. (10), where a microphotograph of a system with nonspherical oriented cells is shown, as well as a dependence of a relative diffusive permeability is stretching. This can be related with both the above-discussed phenomena.

Let us consider a flow in the vertical direction, as it is shown in Figure 5a. Streamlines are shown in the scheme, as well as a vertical cross-section on the cutting surface that coincides with the streamlines. To simplify the approach, we will use the cross-section in the point of the symmetry (see Figure 5a).

This assumption provides a simple procedure for 2-dimensional tortuosity $T^{\prime}$ ' estimation. By considering that tortuosity as the ratio between the effective streamline pathway and the minimal pathway length (13), then the tortuosity caused by the cell contour is given by

$$
\mathrm{T}^{\prime}=1+\frac{\mathrm{d}}{\mathrm{l}^{\prime}}
$$

It is also possible to make a simplified estimation of the tortuosity pathway around the cylinder inscribed in the prism (Figure 5b). Replacing a trajectory around an ellipsoid by the line $\overline{A B C}$, with a point $B$ located on a distance $\overline{\mathrm{OB}}=\mathrm{d} / 2$, we obtain a simple geometrical approximation:

$$
T^{\prime}=\frac{1}{\cos (\varphi)}=\frac{\overline{A B}}{\overline{A O}}=\sqrt{1+\frac{d^{2}}{\left(I^{\prime}\right)^{2}}}
$$

Transition from 2-D to 3-D Tortuosity. Transition from two-dimensional simulation of porous media to the three-dimensional more real situation may be made through a procedure based on the assumption of fractal properties of porous media. Fractal porous media often occur in nature (14-16). As mentioned by Vidales and Miranda (16) for the case of $T=L_{e} / L_{0}$ the channel length of a random walk tends to a fractal walk as $L_{e} \sim r^{D}$. Here $L_{e}$ is the channel length, $L_{0}$ is the porous layer thickness or the path length in the empty space, $r$ is the linear dimension of the walking area, and $D$ is the fractal dimension.

It is possible now to define the 3-D tortuosity as $\mathrm{T}_{3 \mathrm{D}}=$ $r^{D} / r^{2}=r^{D-2}$ and 2-D tortuosity as $T_{2 D}=r^{D^{\prime}} / r=r^{D^{\prime}-1}$, where $D$ and $D^{\prime}$ are the fractal dimensions of 3-D and 2-D channels, respectively. If in both cases the linear dimension $r$ is the same, then $r \approx T_{3 D}^{1 /(D-2)}=T_{2 D}^{1 /\left(D^{\prime}-1\right)}$, and hence, $T_{3 D}=\left(T_{2 D}\right)^{(D-2) /\left(D^{\prime}-1\right)}=T_{2 D}^{m}$. This means that, as long as we know the value for m, the 3-D tortuosity may be estimated from the 2-D tortuosity.

Let us now see how $m=(D-2) /\left(D^{\prime}-1\right)$ can be estimated. From the computer image simulation of 2-D porous media of a binary mixture with particles of large size ratio 15.75 , as well as for ternary mixtures, a simulation procedure presented in a previous work (17, 18), the fractal dimension of 2-D channels network was measured as $D^{\prime} \approx 1.3$. The value of 3-D fractal dimension was borrowed from Zosimov and Lyamshev (19) as being, on average, $\mathrm{D} \approx 2.45$. Therefore, $\mathrm{m} \approx 0.45 / 0.3=1.5$.

When $D-1=D^{\prime}$ the tortuosity is constant $T_{3 D}=T_{2 D}$. It must be pointed out that a transitional value $\mathrm{m}$ is sensitive to measurements and may be used only as an estimate. If we postulate that the 3-D channel network fractal dimension cannot be less than $D^{\prime}$ then $m \geq 1.0$. 


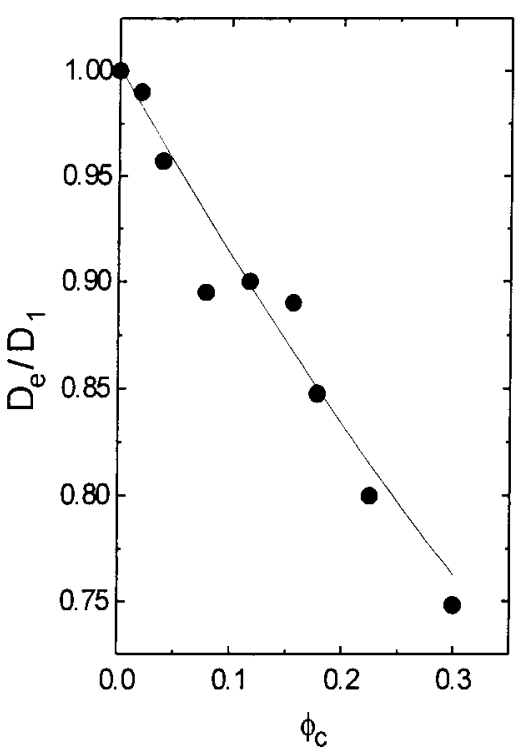

(a)



(b)



(c)

Figure 8. Comparison of experimental data of $D_{0} / D_{1}$ for oxygen diffusion in fermentation media by $\mathrm{Ho}$ and $\mathrm{J} u$ (1988) and proposed model: (a) S. cerevisiae; (b) E. coli; (c) P. Chrysogenum. Points are experimental data. Lines are the prediction by eq 20.

More detailed analysis of this problem will be devoted in a special article.

For the conversion from 2-dimensional tortuosity $T^{\prime}$, to three-dimensional tortuosity $\mathrm{T}$ we can apply the transmission function $T=\left(T^{\prime}\right)^{3 / 2}$. Therefore, for the prism we obtain

$$
\mathrm{T}=\left(1+\frac{\mathrm{d}}{\mathrm{I}^{\prime}}\right)^{3 / 2}
$$

and, for the cylinder approach, we will have

$$
T=\left(1+\frac{d^{2}}{\left.(I)^{\prime}\right)^{2}}\right)^{3 / 4}
$$

The maximum tortuosity corresponding to the horizontal position of the prism or of the cylinder is equal, respectively, to 2.83 and 1.685 . These values lie in the range of tortuosity of particle packed beds. The minimal tortuosity for a vertical position of the prism and of the cylinder is $T=(1-1 / n)^{3 / 2}$ and $T=\left(1+1 / n^{2}\right)^{3 / 4}$, respectively. As we can see, the tortuosity $T \rightarrow 1.0$ with increasing values for the dimensionless length $\mathrm{n}$.

Now, it is possible to estimate a value of $\beta$ for tortuosity $\mathrm{T}_{\mathrm{c}}=1 / \epsilon^{\beta}$ using eq 18b, cylinder approach, and the data by $\mathrm{Yu}$ et al. (20) for a mono-sized cylinder layer with different $\mathrm{n}$. Data obtained by $\mathrm{Yu}$ et al. (20) can be approximated, in the range of $\mathrm{n}$ up to 15.5 , by a linear function (19) with a correlation coefficient 0.9968:

$$
\epsilon=0.3053+0.02557 n
$$

We may calculate the average tortuosity from eq 18 for homogeneous cell distribution and the porosity $\epsilon$ from eq 19. Then, we can estimate $\beta=-\ln (T) / \ln (\epsilon)$ as follows: for $\mathrm{n}=1.5, \beta=0.4$; for $\mathrm{n}=2, \beta=0.338$; for $\mathrm{n}=3, \beta=$ 0.284 , and for $\mathrm{n}=4, \beta=0.259$. In addition, it must be pointed out that, from Yu et al. (20), the effect of packing density and cel Is length distribution must be taken into consideration (see Table 1). Table 1 contains data calculated on the basis of $\beta$ determined above for a mono-sized cylinder pack $\left(\epsilon_{\mathrm{m}}\right.$ and $\left.\mathrm{T}_{\mathrm{m}}\right)$, as well as for a loose and dense

\begin{tabular}{|c|c|c|c|c|c|c|}
\hline$n$ & $\begin{array}{c}\epsilon_{\mathrm{m}} \\
\text { monosized }\end{array}$ & $\begin{array}{c}\epsilon_{\mid} \text {loose } \\
\text { packing }\end{array}$ & $\begin{array}{l}\epsilon_{\mathrm{d}} \text { dense } \\
\text { packing }\end{array}$ & $\begin{array}{c}\mathrm{T}_{\mathrm{m}} \\
\text { monosized }\end{array}$ & $\begin{array}{l}\mathrm{T}_{1} \text { loose } \\
\text { packing }\end{array}$ & $\begin{array}{l}\mathrm{T}_{\mathrm{d}} \text { dense } \\
\text { packing }\end{array}$ \\
\hline & & 0.45 & 0375 & 1.528 & 1.376 & \\
\hline & & & & & & \\
\hline & & & & & & \\
\hline & 0.408 & 0.5 & 0.38 & 1.262 & 1.2 & 1.285 \\
\hline
\end{tabular}

Table 1. Dependence of Average Tortuosity on $\mathbf{n}$ and on Type of Packing of Layers

Table 2. Examples of Calculation for the Model (Eq 20) to Fit the Data from Ho and J u (12)

\begin{tabular}{llcccll}
\hline microorganism & $\mathrm{n}$ & \multicolumn{1}{c}{$\beta$} & $\alpha$ & $\phi_{\mathrm{c}}$ & $\mathrm{a}\left(\epsilon_{0}^{\mathrm{S}} / \epsilon_{\mathrm{av}}\right)$ & $\mathrm{D}_{\mathrm{e}} / \mathrm{D}_{\mathrm{I}}$ \\
\hline S. cerevisiae & & & & 0.1 & 1.0546 & 0.91 \\
& 1.5 & 0.4 & 1.4 & 0.2 & 1.134 & 0.83 \\
& & & & 0.3 & 1.259 & 0.764 \\
E. coli & 4 & 0.259 & 1.259 & 0.1 & 1.03 & 0.902 \\
P. chrysogenum & 4 & 0.259 & 1.259 & 0.1 & 1.0 & 0.876
\end{tabular}

packing of cylinders with log-normal length distribution $\left(\epsilon_{1}\right.$ and $\left.T_{1}\right)$ and $\left(\epsilon_{d}\right.$ and $\left.T_{d}\right)$, respectively.

Finally, the approach developed above was applied to data presented by $\mathrm{Ho}$ and $\mathrm{J} u$ (12), who reported high values for the ratio $D_{e} / D_{1}$ where $D_{1}$ is the oxygen diffusion coefficient in a fermentation medium containing $0.5 \mathrm{wt}$ $\%$ of sodium carboxymethylcellulose for preventing cell sedimentation (see Figure 8). Three types of microorganisms were investigated. For the interpretation of the experimental data, we used the information on cell sizes referred by the authors. thus, for S. cerevisiae, we used $5 \times 8 \mu \mathrm{m}$ (assumed $\mathrm{n}=1.5$ ); for $\mathrm{E}$. coli, $0.5 \times 2 \mu \mathrm{m}(\mathrm{n}=$ 4), and P. chrysogenum was considered as a long rod (we assumed $n=4)$. For the calculations shown in Table 2 , eq 16 under the form of eq 20 was used together with the graphs of Figure $8 \mathrm{~b}$ for $\mathrm{a}\left(\epsilon_{0}^{\mathrm{S}} / \epsilon_{\mathrm{av}}\right)$

$$
\mathrm{D}_{\mathrm{e}} / \mathrm{D}_{1}=\mathrm{a}\left(\epsilon_{0}^{\mathrm{S}} / \epsilon_{\mathrm{av}}\right)\left(1-\phi_{\mathrm{c}}\right)^{\alpha}
$$

where $\alpha=1+\beta$. Results of calculations based on the developed approach are shown in Table 2.

The model curves for each microorganism, together with the experimental data from $\mathrm{Ho}$ and $\mathrm{J} \mathrm{u}$, are pre sented in Figure 8. It is clear that the model fits well 


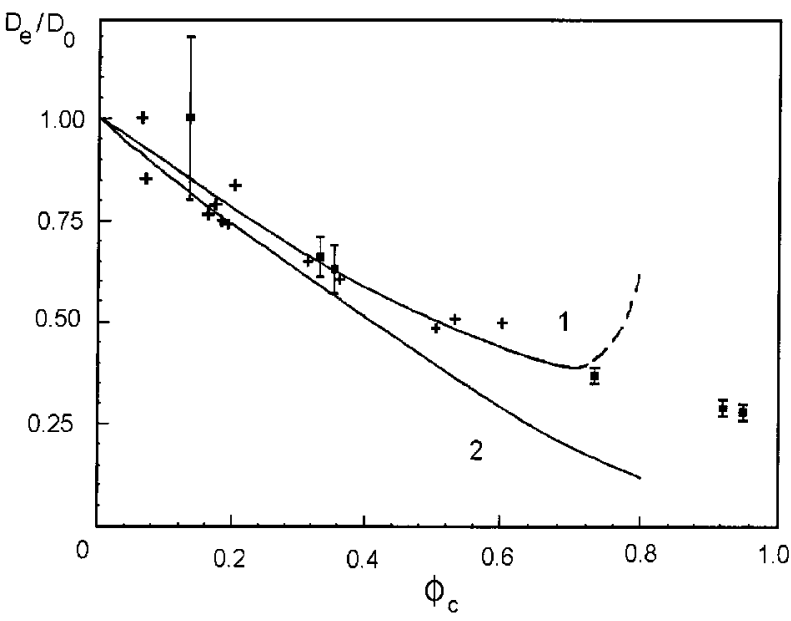

Figure 9. Comparison of Libicki et al. (1988) data for diffusion of oxygen in natural cell aggregates (square points) and artificial cell aggregates (cross points) with proposed model prediction. Line 1 is calculation by formula $D_{e} / D_{0}=a\left(\epsilon_{0} / \epsilon_{a v}\right)\left(1-\phi_{c}\right)^{1.338}$ and line 2 shows the tortuosity effect only: $D_{e} / D_{0}=\left(1-\phi_{c}\right)^{\alpha}$.

into the experimental data taken from microorganisms with substantial differences in shape.

Another example is shown in Figure 9, where data on oxygen diffusion in natural cell aggregates (square points) and artificial cell aggregates (cross points), re ported by Libicki et al. (10), are presented. The proposed model prediction is presented as well. From a microphotograph presented in that article, the parameters $\mathrm{n}=2$ $(\beta=1.338)$ were determined. The range of cell axis orientation was determined to be $0-\pi / 4$. Line 1 represents the calculation by formula $\mathrm{D}_{\mathrm{e}} / \mathrm{D}_{0}=\mathrm{a}\left(\epsilon_{0} / \epsilon_{\mathrm{av}}\right)(1$ $\left.-\phi_{c}\right)^{1.338}$ with the correction from Figure $7 \mathrm{~b}$. Line 2 shows the tortuosity effect, without using the correction function $\mathrm{a}\left(\epsilon_{0}^{\mathrm{S}} / \epsilon_{\mathrm{av}}\right): \mathrm{D}_{\mathrm{e}} / \mathrm{D}_{0}=\left(1-\phi_{c}\right)^{\alpha}$. As was mentioned above, the model has limitations in the zone of large cell volume fractions, which might be related with the cell overlapping effect.

The goodness of fit of the model described by line 1 proves that shape and orientation of cells must be considered in nonhomogeneous gels. As we can see, even for simplified models, the relationship between system parameters with immobilized cells is complex. The average volume fraction is a function of the layers thickness, and $\alpha$ is also a function of layers thickness, of the cell shape, of the cell distribution between the layers, and of the cell orientation inside the gel matrix.

Although only a reduced number of experimental results was used to val idate type 2 nonhomogeneity, the presented information is enough to confirm that the developed approach is useful and can explain observed anomalous low and high values of diffusivity obtained in some immobilized cell systems.

\section{Conclusion}

As shown by the analysis of published data, not all experimental data can be described using a simple homogeneous random pore model. For the random pore model, where the conventional order value for $\alpha$ is 2, the range of possible variation may be $\alpha=1.8-2.25$. A random pore model must describe all data that lie outside this range with a nonhomogeneous cells distribution inside the gel.

As was shown, two main types of nonhomogeneous cell distribution can be considered: (1) nonhomogeneous cells or cells cluster distribution in gel when $\alpha>2.25$ (type 1) and (2) nonhomogeneity related with anisotropy of cells space orientation when $\alpha<1.8$ (type 2).

For the nonhomogeneity of type 1 , the cell volume fraction in the layer or layers occupied by cells must be considered instead of the average cell volume fraction in the gel.

For the nonhomogeneity of type 2, the average crosssection free area available for diffusion does not coincide with the cross-section free area calculated as the average value, especially for high cell loadings. Tortuosity of such a system will be smaller than it would be if it was obtained with a spherical cell approach.

The approach presented in this work may be useful for modeling and analysis of bioreactors whenever the conventional random pore model for diffusion is not valid.

Furthermore, this work puts in evidence the absence of reliable information on the phenomena associated with the use of immobilized cell systems, clearly demonstrating that much work needs to be done on the characterization of the transport properties in these systems and its relation with biomass physiology and activity.

\section{Acknowledgment}

The authors thank the NATO Science Fellowships Program (Portugal) for the fellowship granted to Dr. Yelshin, which enabled the accomplishment of the present work.

\section{Notation}

$D_{c} \quad$ diffusion coefficient through cells

$D_{e} \quad$ coefficient of effective diffusion in gel with immobilized cells, $\mathrm{cm}^{2} / \mathrm{s}$

$D_{g} \quad$ coefficient of effective diffusion in pure gel, $\mathrm{cm}^{2} / \mathrm{s}$

$D_{g}{ }^{*} \quad$ diffusion coefficient in parts of gel of cellcontaining gel not occupied by cells

$\begin{array}{ll}D_{0} & \text { coefficient of diffusion in bulk liquid, } \mathrm{cm}^{2} / \mathrm{s} \\ D_{1} & \text { oxygen diffusion coefficient in fermentation }\end{array}$

I medium

d cell diameter or an approximating cylinder diameter

h length of the cylinder or cell

$\mathrm{f}_{\mathrm{pr}} \quad$ cross-section area of the cylinder or prism located in the center of symmetry

$k \quad$ mass transfer coefficient

$k_{1}$ and $k_{2} \quad$ mass transfer coefficients in the gel layers 1 and 2

$\mathrm{L}_{1}$ and $\mathrm{L}_{2} \quad$ thickness of the layer 1 and layer 2

$\mathrm{n}=\mathrm{h} / \mathrm{d} \quad$ dimensionless length of the cylinder or prism

T tortuosity

$\mathrm{T}_{\mathrm{c}} \quad$ tortuosity created by cells

$T_{c 1}$ and $T_{c 2}$ tortuosity created by cells in the gel layers 1 and 2

$\mathrm{T}_{\mathrm{g}} \quad$ the tortuosity of gel matrix

$\alpha \quad$ order value in eq 2

$\delta \quad \mathrm{L}_{1} / \mathrm{L}$ dimensionless thickness

$\epsilon_{\mathrm{c}} \quad$ average porosity of two layers system

$\epsilon_{\mathrm{c} 1}$ and $\epsilon_{\mathrm{c} 2}$ porosity of gel layers 1 and 2

$\epsilon_{\mathrm{g}} \quad=1-\phi_{\mathrm{p}}$ gel matrix porosity

$\phi_{c} \quad$ cell volume fraction for homogeneous cell distribution in the gel matrix

$\phi_{\mathrm{c} 1}$ and $\phi_{\mathrm{c} 2}$ cells volume fraction in the layers 1 and 2

$\phi_{\mathrm{p}} \quad$ polymer volume fraction in the gel

$\eta \quad=\mathrm{D}_{\mathrm{e}} / \mathrm{D}_{0}$ overall diffusivity coefficient

$\eta_{\mathrm{g}} \quad=\mathrm{D}_{\mathrm{g}} / \mathrm{D}_{0}$ partial diffusivity coefficient in pure gel 
$\eta_{\mathrm{c}} \quad=\mathrm{D}_{\mathrm{e}} / \mathrm{D}_{\mathrm{g}}$ partial diffusivity coefficient in immobilized cells

$\eta_{\mathrm{cl}} \quad=\mathrm{D}_{\mathrm{el}} / \mathrm{D}_{\mathrm{g}}=\epsilon_{\mathrm{cl}} / \mathrm{T}_{\mathrm{cl}}$ diffusivity coefficient in gel layer 1

$\eta_{\mathrm{c} 2}=\mathrm{D}_{\mathrm{e} 2} / \mathrm{D}_{\mathrm{g}}=\epsilon_{\mathrm{c} 2} / \mathrm{T}_{\mathrm{c} 2}$ diffusivity coefficient in gel layer 2

$\theta$

angle between the vertical position and the cell axis

\section{Subscripts}

1

2

high cell concentration layer

low cell concentration layer

\section{References and Notes}

(1) Mota, M.; Teixeira, J . A.; Yelshin, A. I mmobilized Particles in Gel Matrix-Type Porous Media. Homogeneous Porous Media Model. Biotechnol. Prog. 2001, 17, 860-865.

(2) Behie, L. A.; Gaucher, G. M. The Application of Continuous Three Phase Fluidized Bed Bioreactor to the Production of Pharmaceuticals. In Bioreactor Immobilized Enzymes and Cells Fundamentals and Applications; Moo-Young, M., Ed.; Elsevier: New York, 1988; pp 197-212.

(3) Arnaud, J . P.; Lacroix, C. Diffusion of Lactose in $\kappa$-Carrageenan/Locust Bean Gum Gel Beads with or without Entrapped Growing Lactic Acid Bacteria. Biotechnol. Bioeng. 1991, 38, 1041-1049.

(4) De Backer, L.; Devleminck, S.; Willaert, R.; Baron, G. Reaction and Diffusion in a Gel Membrane Reactor Containing I mmobilized Cells. Biotechnol. Bioeng. 1992, 40, 322328.

(5) Hannoun, B. J .; Stephanopoulos, G. Diffusion Coefficient of Glucose and Ethanol in Cell-Free and Cell-Occupied Calcium Alginate Membranes. Biotechnol. Bioeng. 1986, 28, 829-835.

(6) Westrin, B. A.; Axelsson, A. Diffusion in Gels Containing Immobilized Cells: A Critical Review. Biotechnol. Bioeng. 1991, 38, 439-446.

(7) Korgel, B. A.; Rotem, A.; Monbouquette, H. G. Effective Diffusion of Calactose in Calcium Alginate Gels Containing I mmobilized Zymomonas mobilis. Biotechnol. Prog. 1992, 8, $111-117$.
(8) Riley, M. R.; Muzzio, F. J .; Buettner, H. M. Diffusion in Heterogeneous Media: Application to Immobilized cell systems. Al ChE J . 1995, 41, 691-700.

(9) Riley, M. R.; Muzzio, F. J .; Buettner, H. M.; Reyes, S. C. A Simple Correlation for Predicting Effective Diffusion in I mmobilized Cell Systems. Biotechnol . Bioeng. 1996, 49, 223227.

(10) Libicki, S. B.; Salmon, P. M.; Robertson, C. R. The Effective Diffusive Permeability of a Nonreacting Solute in Microbial Cell Aggregates. Biotechnol. Bioeng. 1988, 32, 68-85.

(11) Chresand, T.J .; Dale, B. E.; Hanson, S. L.; Gillies, R. J . A Stirred Bath Technique for Diffusivity Measurements in Cell Matrices. Biotechnol. Bioeng. 1988, 32, 1029-1036.

(12) Ho, C. S.; J U, L.-K. Effects of Microorganisms on Effective Oxygen Diffusion Coefficient and Solubilities in Fermentation Media. Biotechnol. Bioeng. 1988, 32, 313-325.

(13) Bear, J . Dynamics of Fluids in Porous Media; Dover Pub.: New York, 1972.

(14) Adler, P. M. Porous Media: Geometry and Transports; Butterworth-Heinemann: Stoneham (USA), 1992.

(15) Logan, B. E.; Kilps, J . R. Fractal Dimensions of Aggregates Formed in Different Fluid Mechanical Environments. Water Res. 1995, 29, 443-453.

(16) Vidales, A. M.; Miranda, E. N. Fractal Porous Media: Relations Between Macroscopic Properties. Chaos, Solitons Fractals 1996, 7, 1365-1369.

(17) Mota, M.; Teixeira, J . A.; Yelshin, A. Image Analysis of Packed Beds of Spherical Particles of Different Sizes. Sep. Purif. Technol. 1999, 15, 59-68.

(18) Mota, M.; Teixeira, J . A.; Yelshin, A. I mage Modelling of Mixed Granular Porous Media. Fluid/Part. Sep. J . 1999, 12, 71-79.

(19) Zosimov, V. V.; Lyamshev, L. M. Fractals in Wave Processes. Uspekhi Fizicheskikh Nauk (Advances in Physic Sciences) 1995, 165, 361-401.

(20) Yu, A. B.; Zou, R. P.; Standish, N. Modifying the Linear Packing Model for Predicting the Porosity of Nonspherical Particle Mixtures. Ind. Eng. Chem. Res. 1996, 35, 3730-3741.

Accepted for publication March 27, 2002.

BP020046R 